\title{
Planejamento de Anéis Unidirecionais em Telecomunicações: uma Aplicação do Método GRASP
}

P.R.A. LOPES ${ }^{1}$ IM-NCE-UFRJ, Cx.P. 68530, 21945-970 Rio de Janeiro, RJ, Brasil.

M. FAMPA'2, IM-COPPE-UFRJ, Cx.P. 68511, 21945-970 Rio de Janeiro, RJ, Brasil.

S. BINATO ${ }^{3}$ PSR Consultoria, Rua Voluntários da Pátria 45/1307, 22270000 Rio de Janeiro, RJ, Brasil.

Resumo. É grande o número de problemas de otimização de origem no setor de telecomunicações. Neste trabalho iremos otimizar um problema de alocação de equipamentos que visa satisfazer demandas a um custo mínimo usando anéis unidirecionais, equipamentos Add Drop Mutiplexer (ADM) em uma rede de telecomunicações. Para resolução deste problema consideraremos a metaheuristica GRASP.

\section{Introdução}

Por Telecomunicações entende-se um conjunto de dispositivos e técnicas para a transmissão de informações instantâneas a longa distância. Essa transmissão pode ser de voz, sinais gráficos, dados, imagens ou sinais de televisão. Todos eles têm os mesmos princípios fundamentais, mas diferem na forma de manipular as informações e nos meios utilizados para transmiti-las.

Problemas de otimização combinatória são abundantes na indústria de telecomunicações. Nestes problemas, dado um conjunto finito de soluções viáveis $X$ e uma função real $f: X \rightarrow \Re$, procuramos uma solução $x^{*} \in X$ onde $f\left(x^{*}\right) \leq f(x), \forall x \in$ $X$.

Teoricamente é possível encontrar uma solução ótima para um problema de otimização combinatória utilizando enumeração, porém na prática isto em geral se torna inviável, pois o número de possíveis soluções cresce exponencialmente com o tamanho do problema.

Grande parte dos problemas de otimização combinatória são intratáveis por natureza ou são grandes o suficiente para tornar inviável o uso de algoritmos exa-

\footnotetext{
${ }^{1}$ patricia@posgrad.nce.ufrj.br

2 orientadora, fampa@cos.ufrj.br

${ }^{3}$ co-orientador, silvio@binato.com.br
} 
tos. Nestes casos, métodos heurísticos são usualmente empregados para achar boas soluções mas não necessariamente garantem a solução ótima.

Muitos trabalhos ligados ao planejamento de redes de telecomunicação foram desenvolvidos nas últimas cinco décadas, os quais diferem entre si por suas características e técnicas de resolução. Podemos citar o artigo [4], o qual descreve quatro temas diferentes e estes utilizam a meta-heurística GRASP. Em particular tratase o problema de planejamento de uma rede SONET, que utiliza um modelo de programação inteira para um problema de planejamento de rede, uma abordagem heurística usada para encontrar soluções aproximadas do modelo inteiro e um problema pequeno é usado para ilustrar o procedimento. Podemos citar também o artigo [6] que faz uso da técnica de geração de coluna e de uma heurística que utiliza basicamente os conceitos de ciclos e anéis para resolver o problema de dimensionamento da rede.

Neste trabalho propomos a meta-heurística GRASP para resolver o problema de planejamento da expansão de uma rede telecomunicações usando anéis unidirecionais (PAURT). Este problema consiste em determinar onde devem ser instalados equipamentos de transmissão de forma que seja atendida toda a demanda com custo mínimo.

Apresentamos um novo procedimento heurístico que consiste na utilização de uma heurística em dois níveis, onde em um primeiro nível seleciona-se um elemento que é utilizado no segundo nível para a geração da solução propriamente dita alocação dos equipamentos de transmissão de dados nos nós da rede de telecomunicação para formação dos anéis unidirecionais e, conseqüentemente, atendimento das demandas de transmissão de dados.

\section{GRASP, conceitos básicos}

A meta-heurística é uma abordagem multi-start iterativa [2], onde cada iteração GRASP [5] é composta de duas fases: construção e busca local.

A melhor solução achada em todas as iterações é exibida no resultado final. Ilustramos o pseudo código GRASP na Figura 1, onde MaxIter é o número máximo de iterações GRASP e Sem é a semente inicial para o gerador de números pseudo aleatórios.

Em cada iteração, utiliza-se uma função gulosa característica do problema, que mede o benefício de selecionar cada elemento e constroi-se uma lista ordenada com todos os elementos que podem ser adicionados à solução, denominada Lista Restrita de Candidatos (RCL). Para diversos problemas, existem mais de uma função gulosa, portanto, a escolha adequada desta função é muito importante tendo em vista que os resultados do GRASP são extremamente influenciados por esta escolha.

A heurística é considerada adaptativa, pois os benefícios associados a cada elemento são atualizados em cada iteração da fase de construção para refletir as mudanças trazidas pela seleção do elemento anterior. Este componente é responsável pela fuga dos ótimos locais, uma vez que diferentes RCLs são geradas em cada passo da fase de construção do GRASP.

O componente probabilístico do GRASP é caracterizado pela escolha aleatória 


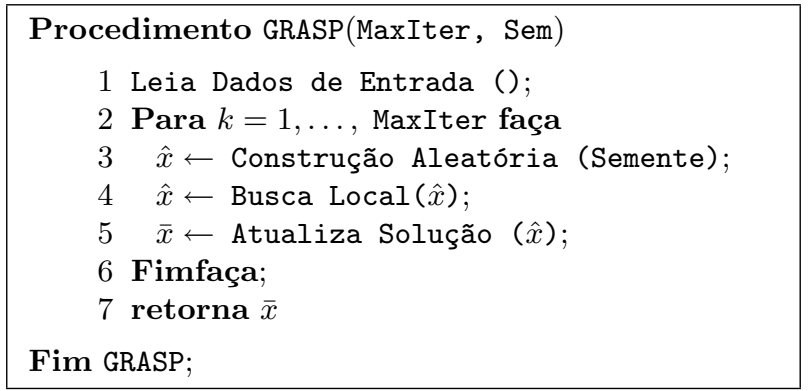

Figura 1: GRASP pseudo código.

de um dos candidatos da RCL, não necessariamente o melhor candidato, diferentemente de um método puramente guloso, na qual o melhor elemento é incluído na solução. Observa-se que a qualidade média da solução depende da qualidade dos membros da RCL e que a diversidade da fase de construção depende diretamente da cardinalidade da mesma. A RCL também é responsável pela fuga dos ótimos locais, pois dependendo da escolha aleatória, elementos diferentes serão incluídos na solução.

Para controlar a qualidade dos elementos da RCL, considere $h_{\min }$ e $h_{\max }$, respectivamente, o menor e o maior valores da função gulosa na fase de construção e $\alpha$ assumindo valores entre zero e um. A RCL é definida em função do pârametro $\alpha$, compreendendo todos aqueles elementos que ainda não fazem parte da solução, cujo impacto no valor da função objetivo esteja no intervalo $\left[h_{\min }, \alpha\left(h_{\max }-h_{\min }\right)+\right.$ $\left.h_{m i n}\right]$. Observa-se que para um problema de minimização $\alpha=0$ implica em uma escolha puramente gulosa enquanto $\alpha=1$, implica em uma escolha aleatória, isto é, para $\alpha=1$, a RCL contém todos os elementos possíveis.

Podemos concluir que o ajuste de $\alpha$ é um dos principais parâmetros a serem sintonizados no GRASP e este pode ser fixo, aleatório ou auto-ajustável (reativo)[3].

Na fase de construção do GRASP, cada elemento da RCL tem, em princípio, a mesma probabilidade de ser selecionado. Entretanto, toda a distribuição de probabilidade pode ser usada atribuindo uma maior probabilidade para seleção de determinados candidatos. Uma maneira de definir uma família de distribuições de probabilidade foi proposta por Bresina [1]. Na sua proposta os elementos candidatos são ordenados de acordo com o valor que lhe é atribuido pela função gulosa e uma função tendência bias(r) é associada ao r-ésimo elemento desta classificação. Diversas funções tendência são introduzidas, como logaritmica $($ bias $(r)=1 / \log (r+$ $1)$ ), linear $(\operatorname{bias}(r)=1 / r)$, polinomial $\left(\operatorname{bias}(r)=1 / r^{n}\right)$, exponencial $(\operatorname{bias}(r)=$ $\left.1 / e^{r}\right)$, aleatória $(\operatorname{bias}(r)=1)$. Uma vez que estes valores foram avaliados para todos os elementos da RCL, a probabilidade de selecionar o elemento $\sigma$ é $p(\sigma)=$ $\operatorname{bias}(r(\sigma)) / \sum_{\sigma \in R C L} \operatorname{bias}(r(\sigma))$. A avaliação da função tendência pode ser usada para restringir os elementos que pertencerão a RCL. Observe que o GRASP padrão utiliza uma função tendência aleatória.

A fase de busca local da solução fornecida pela fase da construção, usa um 
procedimento de enumeração e uma estrutura da vizinhança, onde toda a vizinhança da solução atual é explorada. Se uma solução melhor é visitada, o procedimento de busca local toma esta como a nova solução do problema. O procedimento de busca local termina quando nenhuma solução melhor é encontrada dentro da vizinhança da solução atual. Isto garante que a solução atual é um mínimo local dentro da estrutura de vizinhança usada.

\section{O Problema de Planejamento de Anéis Unidi- recionais em Redes de Telecomunicação}

Consideraremos neste trabalho uma rede de telecomunicação que se distribui em cidades, centros ou regiões. Para cada par de pontos $i$ e $j$ desta rede, define-se um ou mais pacotes de dados $d_{i j}$, que deverão ser transmitidos de $i$ para $j$. A transmissão destes dados na rede pode ser implementada através de uma arquitetura de anel, isto é, procuramos definir todos os subconjuntos de nós que se interligam por fibra ótica que chamaremos de ciclo suporte [7]. Estes atendem a um certo número de dados, onde a união dos subconjuntos atenderá a toda a demanda da rede. Uma vez identificados os ciclos suportes, buscamos construir os anéis, isto é, instalar os equipamentos de transmissão.

A transmissão de dados de um ponto $i$ para um ponto $j$ da rede será então realizada através de um destes anéis, o qual deverá ter equipamentos de transmissão de dados instalados tanto no ponto que envia os dados, como no ponto que os recebe. Observamos que cada pacote de dados deverá ser inteiramente transmitido em um mesmo anel, não podendo ser subdividido. Podemos ter várias demandas relacionadas a um mesmo par de nós e distribuí-las em um ou mais anéis. Para tratar casos como este, consideramos pontos virtuais na rede. Por exemplo, se existem duas demandas entre os nós $i$ e $j$, consideramos uma delas entre os nós $i$ e $j$ e a outra entre os nós virtuais $i^{\prime}$ e $j^{\prime}$, que são na realidade cópias de $i$ e $j$.

Contudo, um nó pode fazer parte de mais de um anel e, conseqüentemente, ter nele equipamentos diferentes, porém estes não se interligam, isto é, em um mesmo anel, todos os equipamentos de transmissão instalados deverão ser do mesmo tipo.

Utilizaremos o equipamento de transmissão $A D D$-DROP Multiplexer (ADM). Este possui tipo, capacidade máxima de transmissão e um custo. A capacidade máxima de transmissão do equipamento utilizado definirá a capacidade de transmissão do anel, a qual independe portanto, do número de equipamentos utilizados. Por outro lado, o custo do anel é dependente do número de equipamentos que foi nele instalado e será dado pela soma dos custos destes equipamentos.

Utilizaremos anéis unidirecionais os quais são compostos pelo o tráfego normal que é transmitido em uma direção em uma fibra e o tráfego de proteção que é transmitido no sentido oposto em outra fibra, garantindo assim que mesmo que haja um rompimento ao longo do caminho, os dados cheguem ao seu destino.

Apresentaremos um modelo de programação linear inteira mista para o problema de planejamento de anéis unidirecionais em rede de telecomunicações. Representaremos no modelo, a rede de transmissão de dados por um grafo $G(V, E)$, onde o conjunto de vértices $V$, representa os pontos da rede de transmissão (cidades, 


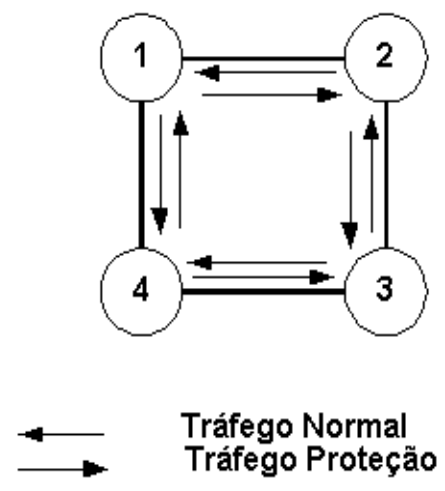

Figura 2: Anel Unidirecional.

centros, etc.) e o conjunto de $\operatorname{arcos} E$ representa as ligações entre os pontos.

No modelo apresentado a seguir (Problema P.P), consideramos todos os ciclos contidos no grafo $G$. Eles representam todas as possíveis estruturas para formação dos anéis por onde os dados serão transmitidos na rede considerada.

Tomemos $\mathcal{C}_{1}, \ldots, \mathcal{C}_{L}$ todos os ciclos do grafo, $\mathcal{C}=\{1,2, \ldots, L\}$ o conjunto dos índices correspondentes e $\mathcal{N}^{l}$ o conjunto de nós que pertencem ao ciclo $\mathcal{C}^{l}$, onde $l \in \mathcal{C}$.

Definimos $p_{k}$ como o preço de cada equipamento utilizado no anel $k, M$ uma constante com valor suficientemente grande, $d_{i j}$ a demanda que vai do nó $i$ para o nó $j, \operatorname{cap}_{k}$ a capacidade do anel $k$ onde a capacidade do anel $k$ é igual a capacidade do equipamento nele utilizado, $n$ o número de vértices do grafo $G, N A$ um limite superior para o número de anéis do problema. A variável binária $e_{i k l}$ assumirá valor um se exite equipamento no nó $i$ do anel $k$ construido no ciclo $l$ e zero caso contrário. A variável $y_{i j k l}$ será um se $d_{i j}$ é atendida pelo anel $k$ construido no ciclo $l$ e zero caso contrário.

A função objetivo representa o gasto total com equipamentos considerando todos os nós, todos os anéis e todos os ciclos. A primeira restrição, estabelece que a demanda total atendida no anel $k$ construido no ciclo $l$, não deve ser maior que sua capacidade. A segunda restrição estabelece que não haverá fluxo partindo de $i$, no anel $k$ construido no ciclo $l$, se não houver equipamento nele instalado, ou seja, se $e_{i k l}=0$. Observe que $M$ deve ser grande o suficiente para tornar esta restrição redundante quando $e_{i k l}=1$. A terceira restrição, estabelece que não haverá fluxo chegando no nó $j$, no anel $k$ construido no ciclo $l$, se não houver equipamento nele instalado. A quarta restrição, estabelece que a demanda $d_{i j}$, só será atendida no anel $k$ construido no ciclo $l$, se existir um equipamento no nó $i$ e no outro nó $j$ neste anel. A quinta restrição estabelece que a demanda $d_{i j}$ só será atendida por um único anel construido em um dos ciclos. 
(P.P) Minimize $\sum_{l \in \mathcal{C}} \sum_{i \in \mathcal{N}^{l}} \sum_{k=1}^{N A} p_{k} e_{i k l}$ sujeito a:

$$
\begin{array}{ll}
\sum_{i, j \in \mathcal{N}^{l}, i \neq j} d_{i j} y_{i j k l} \leq \operatorname{cap}_{k}, & k=1, \ldots, N A, l \in \mathcal{C} \\
\sum_{j \in \mathcal{N}^{l} \backslash i}^{\sum_{i j} y_{i j k l} \leq M e_{i k l},} & k=1, \ldots, N A, i \in \mathcal{N}^{l}, l \in \mathcal{C} \\
\sum_{i \in \mathcal{N}^{l} \backslash j} d_{i j} y_{i j k l} \leq M e_{j k l}, & k=1, \ldots, N A, j \in \mathcal{N}^{l}, l \in \mathcal{C} \\
y_{i j k l}-\frac{e_{i k l}+e_{j k l}}{2} \leq 0, & i, j \in \mathcal{N}^{l} \mid i \neq j, k=1, \ldots, N A, l \in \mathcal{C} \\
\sum_{l \in \mathcal{C}} \sum_{k=1}^{N A} d_{i j} y_{i j k l}=d_{i j}, & i, j \in \mathcal{N} \mid i \neq j, \\
e_{i k l}, y_{i j k l} \in\{0,1\}, & i, j \in \mathcal{N}^{l} \mid i \neq j, k=1, \ldots, N A, l \in \mathcal{C}
\end{array}
$$

Observe que o número dos ciclos em um dado grafo cresce exponencialmente com seu número de nós e o número de variáveis binárias pode se tornar muito grande. Em exemplos reais, este número é freqüentemente muito grande. Como exemplo, consideremos a rede $(\mathrm{G})$ ilustrada na Figura 3 , onde existem três ciclos $\left(l_{1}, l_{2}\right.$ e $\left.l_{3}\right)$.
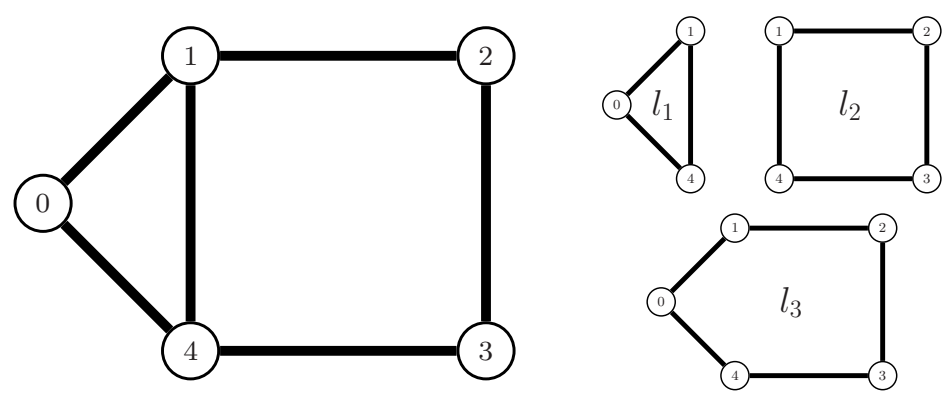

Figura 3: Grafo e Ciclo.

Consideremos 2 tipos de ADM's e três demandas que deverão ser atendidas. Então o número de variáveis binárias será 675 , e o número de restrições será 605 . Se tomarmos 5 ADM's, o número de variáveis binárias passará para 1125 e o número de restrições será 995 .

Concluimos que a utilização de um método de otimização combinatória clássico, como por exemplo branch and bound, tornaria muito difícil a resolução de problemas reais, levando-nos a optar por uma metaheurística que nos forneça uma solução próxima a exata porém com um tempo computacional razoável. 


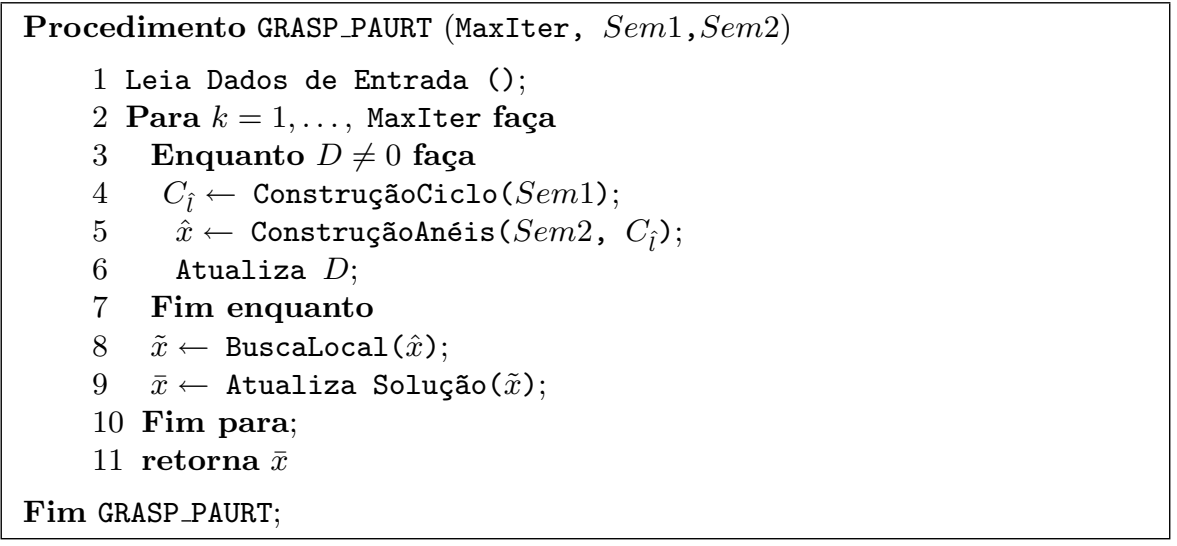

Figura 4: Pseudo código do GRASP para solução de problemas de planejamento de anéis unidirecionais em redes de telecomunicações.

\subsection{Meta-heurística GRASP_PAURT}

Apresentaremos um resumo dos procedimentos de construção e de busca local (GRASP) implementados para obter-se uma solução para o problema PAURT.

Uma solução viável para o problema PAURT consiste basicamente da construção de anéis sobre ciclos de suporte do grafo que representa a rede de transmissão dos dados, para que todas as demandas sejam atendidas. A figura 4 ilustra os passos principais do GRASP para solução de problema de planejamento de anéis unidirecionais em rede de telecomunicações, onde a fase de construção foi dividida em um procedimento construtivo de dois níveis (ConstruçãoCiclo e ConstruçãoAnéis).

O procedimento Leia Dados de Entrada consiste em ler os dados necessários para que seja possível executarmos o GRASP_PAURT. Estes dados, em essência, são o número de nós $(n)$ da rede, o grafo $G=(N, E)$ que representa a rede de telecomunicações, o conjunto de demandas que deverá ser atendida entre cada par de pontos da rede, o conjunto de ciclos de suporte do grafo $G$, gerados previamente, os tipos de equipamentos de transmissão disponíveis, suas capacidades máximas de transmissão e seus custos, o número de iterações do GRASP_PAURT (MaxIter), as sementes para o gerador de números aleatórios (Sem1, Sem2) utilizadas na fase de construção, o parâmetro $\alpha\left(\alpha_{1}, \alpha_{2}\right)$ que determina o tamanho da RCL e o tipo da função tendência utilizada para determinar as chances de cada elemento da RCL.

A próxima etapa, já interna ao loop de iterações da heurística GRASP, é o procedimento construtivo para geração de soluções viáveis para o problema PAURT. Nossa proposta de heurística consiste de um procedimento construtivo dividido em dois níveis, onde no primeiro nível faz-se a seleção de um ciclo de suporte que será utilizado, no segundo nível do procedimento construtivo, para a geração dos anéis, responsáveis pela transmissão dos dados pela rede de telecomunicações. O procedimento construtivo a dois níveis termina somente quando todas as demandas forem atendidas. 
O primeiro nível da heurística construtiva (ConstruçãoCiclo) é responsável pela seleção de um ciclo de suporte. A escolha natural para o ciclo do grafo da rede de transmissão é selecionar o ciclo que suporta o maior número de demandas, ou seja, o ciclo que contém o maior número de nós com demandas. Esta escolha é dita a natural pois ela permite a seleção de anéis com maior capacidade, e menor custo unitário, no segundo nível da heurística construtiva. Logo, escolher ciclos do grafo que suportam o maior número de demandas ainda não atendidas tende a minimizar o custo final de expansão.

Esta medida, o número de demandas não atendidas pertencentes a cada um dos ciclos de suportes, será utilizada como a função "gulosa" $\left(h_{1}().\right)$ do procedimento construtivo do primeiro nível da fase de construção do GRASP_PAURT.

A lista restrita de candidatos (RCL) do primeiro nível é definida a partir do parâmetro $\alpha_{1}\left(0 \leq \alpha_{1} \leq 1\right)$ e dos valores máximos e mínimos, respectivamente $h_{\max }$ e $h_{\min }$, da função "gulosa", ou seja, pertencem a lista restrita de candidatos todos os ciclos cujo número de demandas em seus nós satisfaz $h_{\max } \leq h\left(C_{l}\right) \leq$ $h_{\max }-\alpha_{1}\left(h_{\max }-h_{\min }\right)$.

A seleção do ciclo de suporte é determinística quando assumimos $\alpha_{1}=0$, isto é, garantimos que a RCL conterá somente o ciclo que atende o maior número de demandas. Por outro lado, fazendo $\alpha_{1}=1$ obtemos uma lista restrita de candidatos com todos os elementos possíveis, isto é, todos os ciclos que suportam algum par de demandas por transmissão de dados, então diz-se que esta escolha para o parâmetro $\alpha_{1}$ torna o procedimento construtivo de primeiro nível puramente aleatório.

O próximo nível da fase de construção é o procedimento ConstruçãoAnéis, cujo objetivo é a alocação de equipamentos de transmissão de dados de telecomunicações (ADM's) para a formação de anéis sobre o ciclo de suporte $C_{\hat{l}}$ selecionado no primeiro nível da heurística construtiva.

Observe que, ao fixarmos o ciclo $C_{\hat{l}}$, o problema de planejamento de anéis unidirecionais em redes de telecomunicações, problema P.P, sofrerá basicamente três modificações: a primeira consiste em modificar a quinto conjunto de restrições para relaxar a garantia de atendimento a todas as demandas por transmissão de dados dado que nem todas as demandas pertencem ao ciclo selecionado. A segunda alteração do modelo reformula a garantia de atendimento às demandas para um "incentivo" ao atendidas dessas considerado na função objetivo do problema modificado.

A última modificação feita no problema P.P consiste em fixar o valor de cada uma das variáveis binárias $e_{i k \hat{l}}=[0,1]$, que indicam os nós onde são instalados equipamentos de transmissão de dados. O problema modificado é representado por P.Sim e sua solução é uma avaliação do impacto da instalação de equipamentos de transmissão em um (ou um par) de nós da rede de telecomunicações.

A solução ótima deste problema modificado, quando faz-se $e_{1 k \hat{\imath}}=e_{2 k \hat{l}}=1 \mathrm{e}$ $e_{1 k \hat{l}}=0, \forall i \notin\{0,1\}$ representa uma avaliação do impacto da instalação de equipamentos do tipo $k$ nos nós 1 e 2 . Resolvendo-se este problema para cada equipamento, cada par de nós, temos um conjunto de medidas que avaliam o impacto da instalação dos equipamentos de transmissão de dados em cada um dos nós da rede de telecomunicações. Este conjunto de medidas é utilizado como a função "gulosa" $\left(h_{2}().\right)$ do procedimento construtivo de geração dos anéis (ou segundo nível da fase 
de construção).

$$
\begin{aligned}
& \text { (P. Simp) Minimize } \sum_{i \in \mathcal{N}^{\hat{\imath}}} \sum_{k=1}^{N A} p_{k} e_{i k \hat{\imath}}-w \sum_{k}^{N A} \sum_{i=1}^{n} \sum_{j=1}^{n} y_{i j k \hat{l}} d_{i j} \text {, } \\
& \text { sujeito a: } \\
& \sum_{i, j \in \mathcal{N}^{\hat{\imath}}, i \neq j} d_{i j} y_{i j k \hat{l}} \leq \operatorname{cap}_{k}, \quad k=1, \ldots, N A, \\
& \sum_{i, j \in \mathcal{N}^{\hat{\imath}}, i \neq j} d_{i j} y_{i j k \hat{l}} \leq M e_{i k \hat{l}}, \quad k=1, \ldots, N A, i \in \mathcal{N}^{\hat{\imath}}, \\
& \sum_{i \in \mathcal{N}^{\hat{\imath}} \backslash j}^{j \in \mathcal{N}^{\hat{\imath}} \backslash i} d_{i j} y_{i j k \hat{l}} \leq M e_{j k \hat{l}}, \quad k=1, \ldots, N A, j \in \mathcal{N}^{\hat{\imath}}, \\
& y_{i j k \hat{l}}-\frac{e_{i k \hat{\imath}}+e_{j k \hat{\imath}}}{2} \leq 0, \quad i, j \in \mathcal{N}^{\hat{\imath}} \mid i \neq j, k=1, \ldots, N A \text {, } \\
& \sum_{k=1}^{N A} d_{i j} y_{i j k \hat{l}} \leq d_{i j}, \quad i, j \in \mathcal{N} \mid i \neq j, \\
& e_{i k \hat{l}}, y_{i j k \hat{l}} \in\{0,1\}, \quad i, j \in \mathcal{N}^{\hat{\imath}} \mid i \neq j, k=1, \ldots, N A \text {. }
\end{aligned}
$$

Define-se a lista restrita de candidatos (RCL) do segundo nível do procedimento construtivo em função do parâmetro $\alpha_{2}$ e dos valores máximos e mínimos, resp. $h_{\max }$ e $h_{\min }$, da função "gulosa" para este procedimento - valor da solução do problema P.Simp - com todos os equipamentos alocados nos nós (ou pares de nós) cujo valor da função "gulosa", $h_{2}($.$) satisfazer h_{\min } \leq h_{e_{i k \hat{\imath}}-e_{j k \hat{l}}} \leq h_{\text {min }}-$ $\alpha_{2}\left(h_{\min }-h_{\max }\right)$.

Da mesma forma que no procedimento do primeiro nível, fazendo $\alpha_{2}=0$ torna a alocação dos equipamentos nos nós determinística, ou seja, o equipamento que alocado a um (ou um par de nós) atende a maior quantidade de demanda será selecionado, enquando que fazendo $\alpha_{2}=1$ esta seleção será totalmente aleatória.

O procedimento construtivo de segundo nível deve alocar novos equipamentos de transmissão de dados nos nós até que todas as demandas do ciclo $C_{\hat{l}}$ sejam atendidas, o valor da solução de P.Simp. é menor que zero sempre que alguma demanda for atendida. Quando todas as demandas do ciclo $C_{\hat{l}}$ forem atendidas, deve-se escolher outro ciclo de suporte fazendo-se mais uma iteração do procedimento de primeiro nível, possibilitando que novas alocações (equipamentos em nós) sejam realizadas.

Dada a maneira como foi construído, podemos concluir que o procedimento de construção (a dois níveis) é uma heurística "gulosa" para a obtenção de soluções viáveis para o problema de planejamento de anéis unidirecionais em redes de telecomunicações.

Dada uma solução víável para um problema combinatório, GRASP advoga a realização de uma fase de aprimoramento da solução executando um procedimento de busca local em entorno da solução obtida anteriormente. No caso do problema PAURT, o procedimento de Busca Local tenta reduzir o número de anéis da rede de telecomunicações alocando as demandas desses em outros anéis com capacidade ociosa. A eliminação de um anel faz com que os equipamentos de transmissão que o caracterizam não sejam mais necessários, reduzindo o custo final da solução e aumentando a eficiência da rede unidirecional instalada. 
Tabela 1: ADM's.

\begin{tabular}{||c||c||c||}
\hline \hline ADM & Capacidade & Preço $\left(10^{6} \mathrm{R} \$\right)$ \\
\hline \hline ADM - 1 & $1008 \mathrm{Mbps}$ & 250,00 \\
\hline ADM - 2 & $4032 \mathrm{Mbps}$ & 450,00 \\
\hline \hline
\end{tabular}

A busca por melhoramentos da solução corrente inicia tentando alocar as demandas dos anéis de menor eficiência (relação entre a demanda alocada no anel e sua capacidade máxima). Este procedimento enumera todas as possibilidades de troca, de uma demanda em um anel para outro, e pára somente quando qualquer modificação na configuração dos anéis (face a mudanças na alocação das demandas) não leva a melhoramentos da solução, ou seja, garante-se que a solução corrente é um mínimo local.

\section{Resultados Numéricos}

Com o objetivo de comparar os resultados entre a resolução de um método exato $\left(Z^{*}\right)$ e o GRASP_PAURT $(\bar{Z})$, consideramos um problema-teste utilizando o grafo da Figura 5, com 5 nós, 7 arestas, o qual possui 9 ciclos de suporte. Neste exemplo alocaremos as demandas descritas na figura 6 , utilizando 2 tipos de equipamentos com seus respectivos preços e capacidades hipotéticos representados na Tabela 1.

Obtemos como valor ótimo para o problema P.P, $Z^{*}=2000$. Podemos observar os resultados encontrados pelo GRASP_PAURT $(\bar{Z})$, na Tabela 2 , utilizando $\alpha_{1}=1$, função tendência randômica e linear, após 100 iterações .

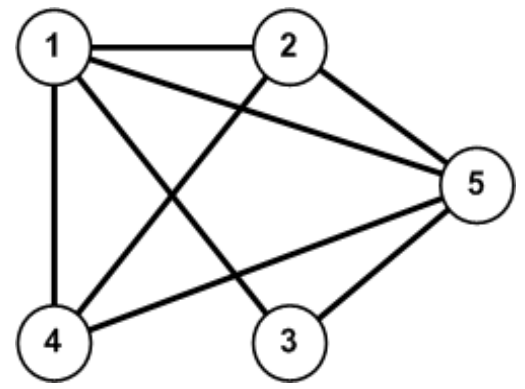

Figura 5: Grafo G

\begin{tabular}{|c|c|c|}
\hline$i$ & $j$ & demanda \\
\hline 5 & 2 & 765 \\
\hline 5 & 4 & 828 \\
\hline 2 & 5 & 268 \\
\hline 3 & 5 & 594 \\
\hline 4 & 5 & 634 \\
\hline
\end{tabular}

Figura 6: Demandas

\section{Conclusões}

Neste trabalho apresentamos um novo procedimento heurístico para a resolução de problemas de planejamento da expansão de redes de telecomunições utilizando anéis 
Tabela 2: Problema Teste.

\begin{tabular}{||c|c|c||}
\hline \hline & Rand $(Z)$ & $\operatorname{Lin}(Z)$ \\
\hline$\alpha_{2}=0,5$ & 2000 & 2000 \\
\hline$\alpha_{2}=0,3$ & 2000 & 2000 \\
\hline \hline
\end{tabular}

unidirecionais. Este novo procedimento heurístico consiste na generalização dos conceitos da meta-heurística GRASP para a resolução deste problema. É importante ressaltar neste processo uma contribuição realizada por este trabalho que foi a utilização de uma heurística em dois níveis, onde em um primeiro nível seleciona-se um elemento que é utilizado no segundo nível para a geração da solução propriamente dita - alocação dos equipamentos de transmissão de dados (ADMs) nos nós da rede de telecomunicação para formação dos anéis unidirecionais e, conseqüentemente, atendimento das demandas de transmissão de dados.

Desenvolvimentos futuros deste trabalho envolvem a implementação de novas funções "gulosas", aperfeiçoamentos na fase de busca local, assim como a pesquisa de meta-heurísticas híbridas, como a hibridização de GRASP com Path Relinking por exemplo.

\begin{abstract}
Combinatorial otimization problems are abundant in telecommunications industry. In this work we will optimize a problem of equipment allocation in order to satisfy demand and to minimize the cost of the network, using unidirectional synchronous rings, equipment Add Drop Mutiplexer (ADM) in telecommunications network. We will use metaheuristica GRASP to solve the problem.
\end{abstract}

\title{
Referências
}

[1] J. Bresina, Heuristic-biased stochastic sampling, em "Proceedings of the thirteenth national conference on artificial intelligence (AAAI-96)", pp. 271-278. American Association for Artificial Intelligence, 1996.

[2] Lin e Kernighan, Randon multi-start local search, 1973.

[3] M.G.C. Resende e C.C. Ribeiro, Greedy randomized adaptive search procedure, AT\&T Labs Research Technical Report, setembro, 2001.

[4] M.G.C. Resende, Combinatorial optimization in telecomunications, AT\&T Labs Research Technical Report, julho, 2001.

[5] M.G.C. Resende, Greedy Randomized Adaptive Search Procedure (GRASP), AT \&T Labs Research Technical Report, dezembro, 1998.

[6] N. Maculan, M.M. Passini, J.A.M. Brito e A. Lisser, "Column Generation Method for Network Design", Kluwer Academic Publishers, Netherlands, 2000.

[7] J.L. Szwarcfiter, Grafor e Algoritmos Computacionais, Editora Campus, pp. 472-484, 1984 
